\title{
The Influential Articles and Title Words in the Journal of Korean Neurological Society Publications between the Years 2016 to 2018
}

\author{
Soo-Bin Im, Moonyoung Chung, ${ }^{1}$ Bum-Tae Kim² \\ Editorial Staff,' Journal of Korean Neurosurgical Society; Department of Neurosurgery, Soonchunhyang University Bucheon Hospital, \\ Bucheon, Korea \\ Editor-in-Chief, Journal of Korean Neurosurgical Society; Department of Neurosurgery, Soonchunhyang University Bucheon Hospital, \\ Bucheon, Korea
}

The Journal of Korean Neurosurgical Society (JKNS) has been recognized as an international scientific journal for more than 10 years. In 2018, the impact factor (IF) of JKNS announced by Journal Citation Reports jumped to 1.187, which was an increase of $38 \%$ compared to the previous year. It is not only the honor of JKNS but also its obligation to direct what the journal should $\mathrm{do}^{2}$. It is clear that readers and other academic parties interested in the journal are already shifting from regional to international platforms, and the editorial committee of JKNS has to reflect on this issue ${ }^{1)}$. In this editorial study, the most cited articles in the journal publications between the years 2016 to 2018 were examined. The six influential articles were selected for capturing the academic interests of international scientific communities. Additionally, we analyzed the titles of the journal publications between the years 2016 to 2018 to identify articles that had received more attention from international readers.

\section{THE INFLUENTIAL ARTICLES}

The citation data was extracted on November 11, 2019. Between January 2016 and November 2018, 314 articles were published in JKNS. The total citation of these articles was 809 and the average citation count per article during the period was 2.58. Articles that were not cited at all were 80 in number, which equates to $25.5 \%$ of all the articles published by the journal. Six most cited articles of each year were considered and the total citation count of these 18 articles was 213. Interestingly, six (33.3\%) of the most cited articles addressed endovascular treatment and the subject matter of five articles among those was clinical studies on mechanical thrombectomy. Another six of the articles were classified into articles of spinal surgery, of which two articles covered studies that carried out spinal endoscopic surgery and another two covered the topic of spinal deformity. Among the most cited 18 articles in the JKNS publications between the years 2016 to 2018, the six influential articles that received the highest international

- Received : December 23, 2019 •Accepted : December 27, 2019

- Address for reprints : Bum-Tae Kim

Department of Neurosurgery, Soonchunhyang University Bucheon Hospital, 170 Jomaru-ro, Bucheon 14584, Korea

Tel : +82-32-621-5289, Fax : +82-32-621-5107, E-mail : bumtkim@gmail.com, ORCID : http://orcid.org/0000-0003-2646-8165

This is an Open Access article distributed under the terms of the Creative Commons Attribution Non-Commercial License (http://creativecommons.org/licenses/by-nc/4.0) which permits unrestricted non-commercial use, distribution, and reproduction in any medium, provided the original work is properly cited. 


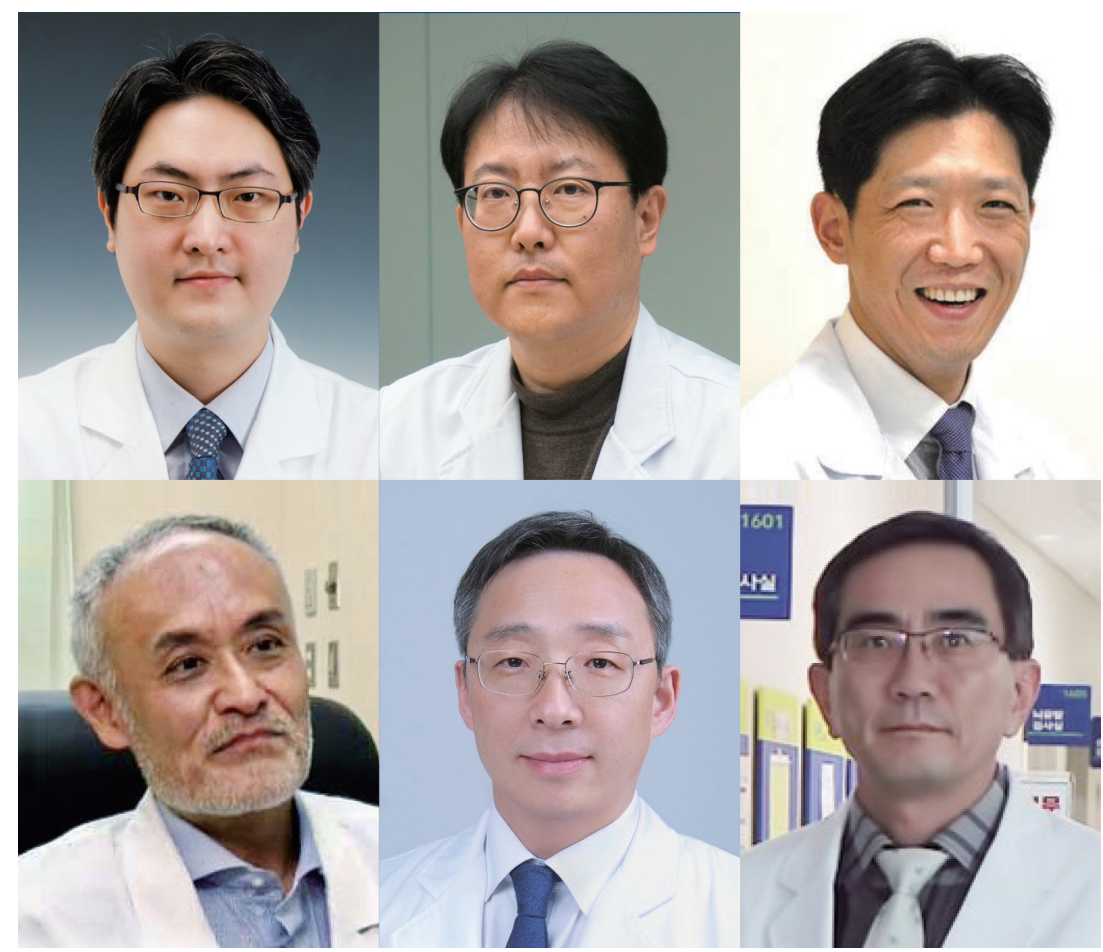

Fig. 1. The corresponding authors of the six influential articles in the Journal of Korean Neurosurgical Society publication between the years 2016 to 2018. From top left to bottom right: Seung-Jae Hyun, Jeong Eun Kim, Chul-Woo Lee, Kenichi Nishiyama, Jaechan Park, and Ki-Bum Sim.

attention and significantly contributed the international growth of the journal were selected. It is our honor to announce these articles with a list of their corresponding authors (Fig. 1).

Choi et al. Effectiveness and Safety of Tranexamic Acid in Spinal Deformity Surgery. 2017;60(1):75-81 by Seung-Jae Hyun of Seoul National University Bundang Hospital.

Cho et al. Korean Clinical Practice Guidelines for Aneurysmal Subarachnoid Hemorrhage. 2018;61(2):127-166 by Jeong Eun Kim of Seoul National University Hospital.

Lee et al. Foraminoplastic Superior Vertebral Notch Approach with Reamers in Percutaneous Endoscopic Lumbar Discectomy: Technical Note and Clinical Outcome in Limited Indications of Percutaneous Endoscopic Lumbar Discectomy. 2016;59(2):172-81 by Chul-Woo Lee of St. Peter's Hospital.

Nishiyama. From Exoscope into the Next Generation. 2017;60(3):289-293 by Kenichi Nishiyama of Niigata Medical Center.

Kang et al. Endovascular Stroke Therapy Focused on Stent Retriever Thrombectomy and Direct Clot Aspiration: Historical Review and Modern Application. 2017;60(3):335-347 by Jaechan Park of Kyungpook National University Hospital.
Jin et al. Development and Growth of the Normal Cranial Vault: An Embryologic Review. 2016;59:192-196 by Ki-Bum Sim of Jeju National University Hospital.

\section{THE MOST CITED TITLE WORDS}

A high citation is an indication that an article was valuable and recognized by the scientific community. Because of its importance, those who manage journals must consider the IF. Writers mostly consider publishing their articles in journals with a high IF; therefore, they are concerned about the frequency with which citations are made from a journal's site. To identify the reason behind the higher citation of some articles over others, words in article titles of 314 articles published in the JKNS between 2016 to 2018 were analyzed with a focus on those that were perceived to be getting more attention from readers.

The citation of an article alone does not necessarily indicate that an article is good. This is because the probability of an article being cited increases with time since its publication. Therefore, a more reliable citation can be obtained by dividing 


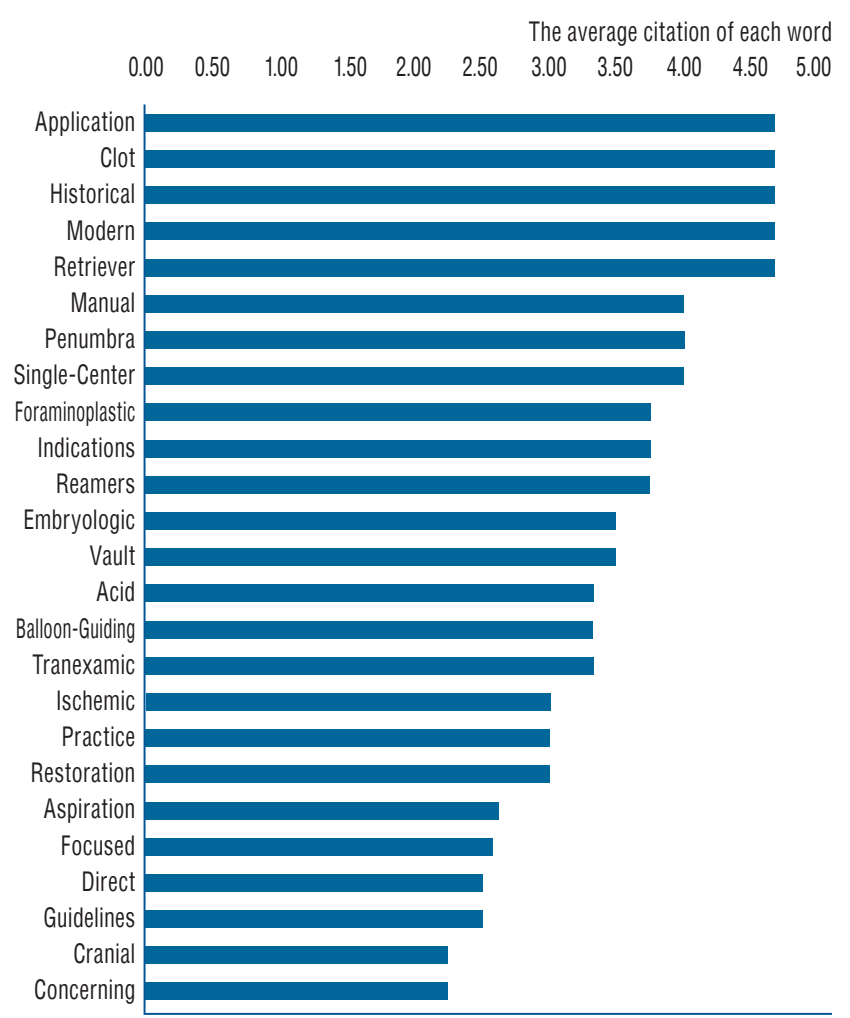

Fig. 2. The top 25 title words with a higher citation.

the citations by the years since publication.

The words in the titles of the selected 314 articles were examined. Title words with no valuable meaning were eliminated. These included numbers, pronouns, personal pronouns, and indefinite articles, such as "Two", "The", "We", "A", and "This". The words with the same meaning that differed based on their plurality were consolidated as one word, e.g., "Factor, Factors $\rightarrow$ Factor" and "metastasis, metastatic, metastasized $\rightarrow$ metastatic". With the remaining words of the titles, the citation of each word was calculated. Then the citation was averaged by the frequency of each word used in the title. As a result, the average citation of each word was obtained. The top 25 title words with a higher citation are shown in Fig. 2.

The title words with the highest value of the average citation per year were "application," "clot," "historical," "modern," and "retriever," which were followed by "manual," "penumbra," "single-center," "foraminoplastic," "indication," and "reamer." With this analysis, words in the titles of articles in the JKNS that are more likely to be cited could be identified. The words associated with a specific surgical procedure or treatment were more likely to be cited, while non-specific anatomical words or broadly-based disease names had a low possibility of being cited. The words which emphasized that the research was latest and/or represented new trends were also more likely to be cited.

\section{CONFLICTS OF INTEREST}

No potential conflict of interest relevant to this article was reported.

\section{AUTHOR CONTRIBUTIONS}

\author{
Conceptualization : BTK \\ Data curation : SBI, MC \\ Formal analysis : SBI, MC, BTK \\ Funding acquisition : BTK \\ Methodology : SBI, MC, BTK \\ Project administration : BTK \\ Visualization : SBI, MC, BTK \\ Writing - original draft : SBI, MC \\ Writing - review \& editing : BTK
}

\section{- Acknowledgements}

This work was supported by Korean Neurosurgical Society and Soonchunhyang University Research Fund.

\section{References}

1. Chung M, Kim BT : Editorial statistics and best reviewers award 2018 for Journal of Korean Neurosurgical Society. J Korean Neurosurg Soc 62 : 1-2, 2019

2. Kim BT, Chung $M$ : Evolution of quality improvement for the Journal of Korean Neurosurgical Society. J Korean Neurosurg Soc 61 : 425426, 2018 